\title{
The timing and route of seminal vesicle dissection during robotic prostatectomy
}

\author{
David I. Lee $\cdot$ Randy Fagin
}

Published online: 3 November 2007

(C) Springer London 2007

\section{Introduction to "Controversies in Robotic Surgery" \\ David I. Lee}

It is with great pleasure that I introduce this new section in the "Journal of Robotic Surgery". This section, which will appear on a regular basis in the journal, will focus on some of the tough questions that surgeons face during their learning curve or application of robotic technologies to surgery. The field of robotic surgery is growing both in experience and technique. As we explore these topics of debate, likely many of these issues have no clear-cut "right" answer, but certainly there are experts who perform similar procedures in markedly different ways, and it is our hope that this section will help the reader gain an understanding of why these surgeons prefer one method over another. We hope that you will gain an appreciation for the struggles and successes of these experiences and techniques and then you can "take home" and incorporate the insights that you find most sensible into your own practice.

In the first in the series of controversies we are pleased to have Dr. Randy Fagin from Austin, TX giving his perspective on dissection of the seminal vesicles at the outset of the robotic prostatectomy, while I will address the later dissection of the seminal vesicles.

D. I. Lee $(\bowtie)$

Division of Urology, Department of Surgery,

University of Pennsylvania School of Medicine,

51 N. 39th St., Suite 266, Philadelphia, PA 19104, USA

e-mail: david.i.lee@uphs.upenn.edu

R. Fagin $(\bowtie)$

The Prostate Center of Austin, Westlake Medical Center, 5656 Bee Caves Rd D-200, Austin, TX 78746, USA

e-mail:rfagin@austin.rr.com

\section{Advantages of the posterior approach to the seminal vesicles during robotic prostatectomy}

Randy Fagin

Although I have had the opportunity to perform laparoscopic and daVinci prostatectomies via both the anterior and posterior approach, I have found the posterior approach to have significant advantages. I use this approach universally and the reasons can be boiled down to two main concepts: access and efficiency.

Access

I believe that the posterior approach more consistently gives the surgeon easy and direct access to the seminal vesicles and vas deferens (SV). Regardless of the size of the patient, the size of their SV, the size of their prostate, or the presence of a median lobe, access to the $\mathrm{SV}$ is never limited in the posterior approach, being located millimeters from the peritoneal surface, while it is often obscured anteriorly. From the posterior approach the SV are always right in front of you, just above the reflection of the peritoneum at the rectum. The ureters are located superolateral to the SV from this vantage point so they should not be at risk of injury during this part of the dissection. For many surgeons the anterior approach, including the dissection of the posterior bladder neck, can be one of the most variable parts of the operation. Depending on variations in the size and shape of the prostate the anterior approach to the SV can be anything from straightforward to highly challenging. Factors like a large gland, a large posterior portion of the gland, protuberant lateral lobes, the presence of a median lobe, and length of the SV can limit access, thus increasing the level of difficulty. These factors may create a situation 
where the surgeon is working in a narrow hole, which may be overcome with more dissection laterally on the bladder pedicles. This maneuver, while widening the working space, may compromise the nerve sparing, carrying the dissection too close to the posterior plate of the neurovascular bundle which lies on the lateral aspect of the prostatovesical junction. In the posterior approach it is easier to stay in the midline away from the laterally located posterior plate of the neurovascular bundle by performing a midline-only dissection at the posterior bladder neck. This is possible because you do not need to create a space sufficient for the complete dissection of the SV. All you need to do is create a midline hole that connects to the space where the SV dissection was completed earlier. Any remaining pedicle tissue can then be easily managed athermally with the placement of a few clips after precise isolation of the neurovascular bundles. Additionally, when performing a nervesparing procedure, athermal dissection lateral to and at the tips of the SV is crucial because of the proximity of the neurovascular bundles. When the SV posterior dissection is performed the large working space of the abdominal cavity makes an athermal dissection straightforward every time.

\section{Efficiency}

Because of the direct access to the SV afforded by the posterior approach, this portion of the procedure consistently takes me $2-4 \mathrm{~min}$. Anatomic variations in the dimensions of the prostate such as large size, protuberant lateral lobes, or a large median lobe do not affect operating time. Even in very obese patients the fourth arm easily retracts the bowel and fat out of the way and once the incision in the peritoneum is made a single assistant can lift the peritoneal flap superiorly, giving perfect exposure of the SV. Additionally, the posterior dissection of the SV allows the posterior bladder neck dissection to merely be a straightforward midline dissection down to the previously created posterior "hole". With the posterior approach your bladder neck dissection is thus simplified, making it more time efficient. You can comfortably peel the prostate off the bladder in the midline until you hit the previously created posterior hole, at which point you just pull the SV up and out.

So for me, the posterior approach not only allows me consistent access to the $\mathrm{SV}$, it also allows me to perform a straightforward and athermal SV dissection in under $5 \mathrm{~min}$ regardless of variations in prostate anatomy. Furthermore, performing the SV dissection via the posterior approach simplifies the posterior bladder neck dissection, keeping me further away from the posterior plate of the neurovascular bundle and improving the time efficiency of that portion of the operation as well.

Having said that, I have watched many talented surgeons perform beautiful anterior dissections of the SV, and although I feel strongly about the advantages of the posterior approach, the outcomes of any approach lie fully in the skill and experience of the surgeon.

\section{The anterior approach to the seminal vesicles during robotic prostatectomy}

\section{David I. Lee}

The anterior approach to the seminal vesicles and the vas deferens (SV) is defined as dissection of these structures after dissection of the bladder neck to gain access to these structures from their anterior surface. The classic approach to laparoscopic prostatectomy as originally described was indeed a posterior approach. The outset of the transperitoneal prostatectomy calls for an incision in the peritoneum through which access to the SV is immediately performed. With the use of the robotic platform for prostatectomy, many surgeons have now utilized the anterior approach. I prefer this approach in my practice for several reasons that I will now describe.

Most importantly, there are no prospective, randomized data showing the superiority of one approach to another, so the choice of approach is mostly personal preference, but I do prefer the anterior approach because I think it is easier. Having performed both techniques, I have seen many cases, especially in patients with excess adipose tissue, where the $\mathrm{SV}$ can be a bit difficult to locate posteriorly. The adipose tissue can obscure the field and make this plane more difficult to find. The posterior approach is impossible for those surgeons who prefer an extraperitoneal approach as well.

Access through the bladder neck is reliable even in patients who are large, as the distance between the bladder and the ampulla of the vas seems to be better preserved and more reliably obtained. Once the ampulla of the vas is identified, the time for dissection of these structures is very reliable and rarely takes more than a few minutes unless the SV are very large. It is also very easy to perform this dissection athermally if this is deemed important by the surgeon. I typically still use bipolar energy judiciously. I believe that with the anterior approach the SV roll upward and away from the neurovascular bundle and thus the risk of nerve injury is lower this way.

Besides the benefits of ease of dissection, there are some theoretical benefits that I believe need further exploration. A recent abstract from the 2007 AUA meeting compared continence data between a high-volume open radical prostatectomist and a similar high-volume laparoscopic prostatectomist at the same institution. The open prostatectomy surgeon had better continence results. What is the likely explanation for this difference? Both surgeons perform an anatomically precise operation. However, one 
major technical difference is that the laparoscopic surgeon uses a transperitoneal posterior approach while the open surgeon uses an extraperitoneal approach. Could it be that there are yet undefined anatomic reasons why incision through the posterior peritoneum somehow adversely affects continence?

Although I do feel the anterior approach is easier for me, it is likely that surgeons will feel more comfortable with whichever approach they have performed more often. Again, no randomized comparative data exist, and although there may be benefits of one technique over the other these are still not realized. Therefore, although I advocate the anterior approach, I believe experience has shown that both approaches are efficient and expedient. 